\title{
Description of a CAD Protocol for the Simulation of Dental Treatment of Amelogenesis Imperfecta
}

\author{
Damien Brezulier*, Julien Lambert and Olivier Sorel \\ Univ Rennes, Pole Odontologie Rennes, France
}

Received: February 27, 2018; Published: March 09, 2018

*Corresponding author: Damien Brezulier, Univ Rennes, CHU Rennes, ISCR, CNRS - UMR 6226, Pole Odontologie F-35000 Rennes, France, Email: damien.brezulier@univ-rennes1.fr

\section{Introduction}

Amelogenesis imperfecta (AI) is an inherited disorder of development that primarily affects dental enamel. The etiology of this pathology is the mutation or the expression alteration of 13 genes [1]. The tissues of both dentitions being reached, the aesthetics of the smile is lost from childhood. The psychosocial impact of AI imposes a relatively young functional and aesthetic care [2]. The difficulty of the reconstruction of tooth tissues in definitive dentition is based on two elements: the associated dento-facial dysmorphosis and the difficulties of bonding due to the alteration of the adhesive bond to the affected enamel. Management with the best prognosis is therefore based on a treatment combining orthodontics and indirect reconstructions [3]. We propose here, the analysis of a case then and the simulation of the treatment by CAD. The interest of this approach is to be able to perform the simulation of the wax-up to offer a coherent therapeutic proposal, both functional and aesthetic.

Initial Situation: (Figures 1 \& 2 ) The patient goes for consultation at the age of 14. He has an amelogenesis imperfecta. Several teeth already had to be extracted for infectious reason. The occlusion is in Class II of Angle with insufficient anterior space to place two maxillary central incisors. During this first therapeutic phase of cleansing, the first maxillary molars are extracted and the anterior teeth are depulped. In a second step, the initial model digital scans are positioned at the correct vertical dimension. The treatment objectives are here the opening of the space of the upper incisors by distaling the lateral sectors in the extraction spaces of the first molars. The mandibular arch will not be treated by orthodontics.

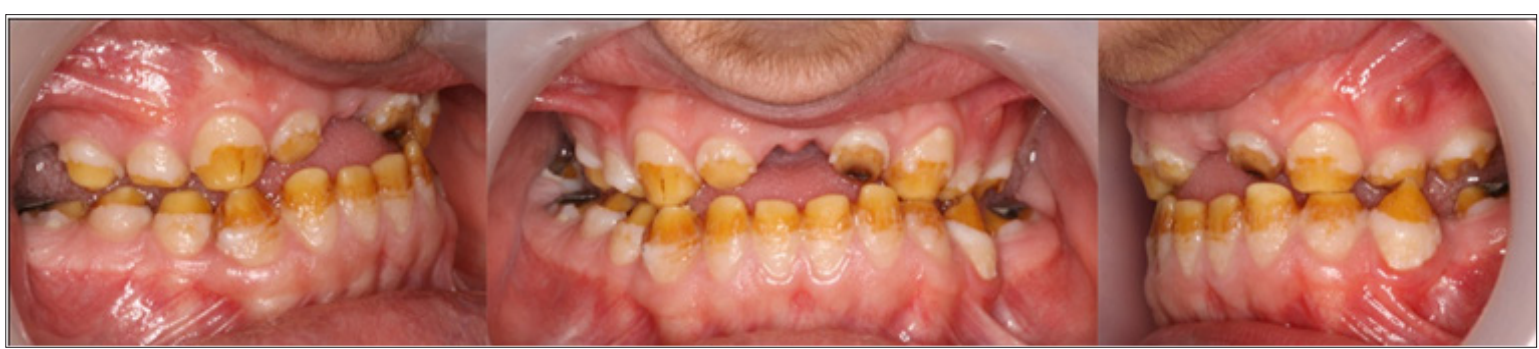

Figure 1: Initial situation, the patient goes for consultation at the age of 14 .

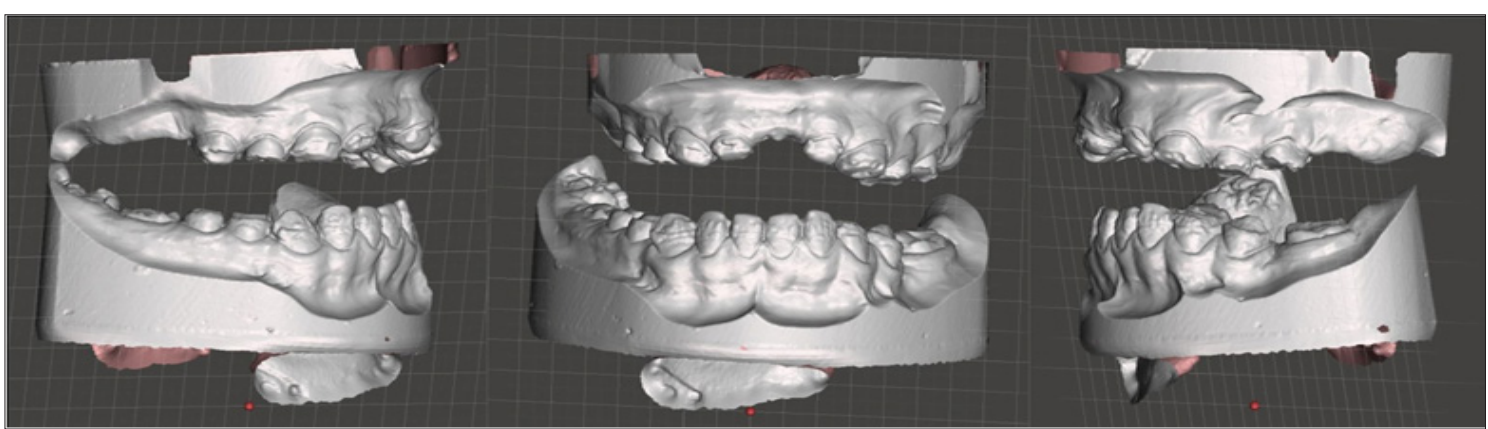

Figure 2: The Patient has an amelogenesis imperfecta. 
Simulation of Reconstruction of Dental Volumes: (Figure 3) this first phase of reconstruction aims to restore teeth to a correct volume. After trimming the teeth away from the collars to avoid the removal of enamel remaining during preparation, the coronal volumes are added. There is no search for occlusion but only a simulation of occlusion plan. It is on this first set of reconstructions that the orthodontic treatment will be conducted.

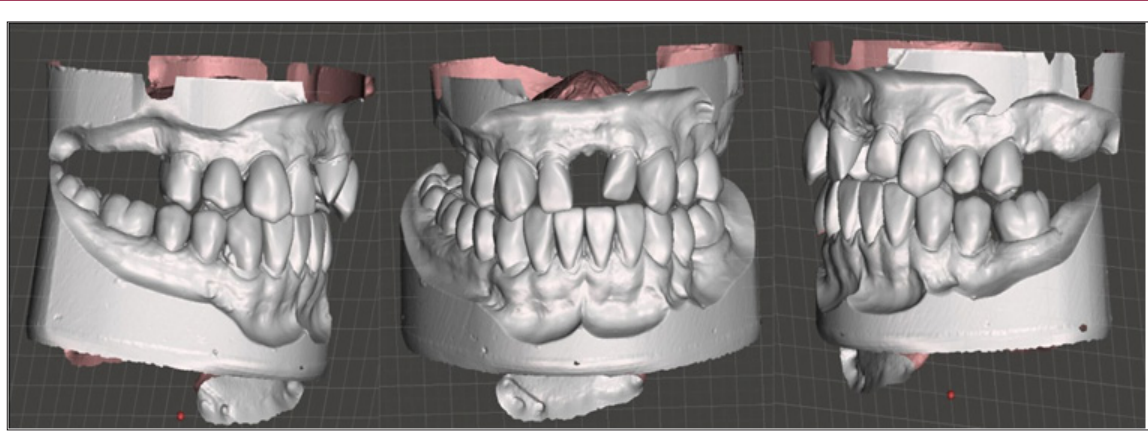

Figure 3: Simulation of reconstruction of dental volumes.

Simulation of Orthodontic Displacement: (Figure 4) the teeth are then individualized and moved. The goal for this patient is to open the anterior space to provide sufficient space for two central incisors. To do this, the lateral sectors are retracted to obtain a Class I occlusion of Angle. At the end of this phase, we have two new models that can be exported to add the missing teeth.

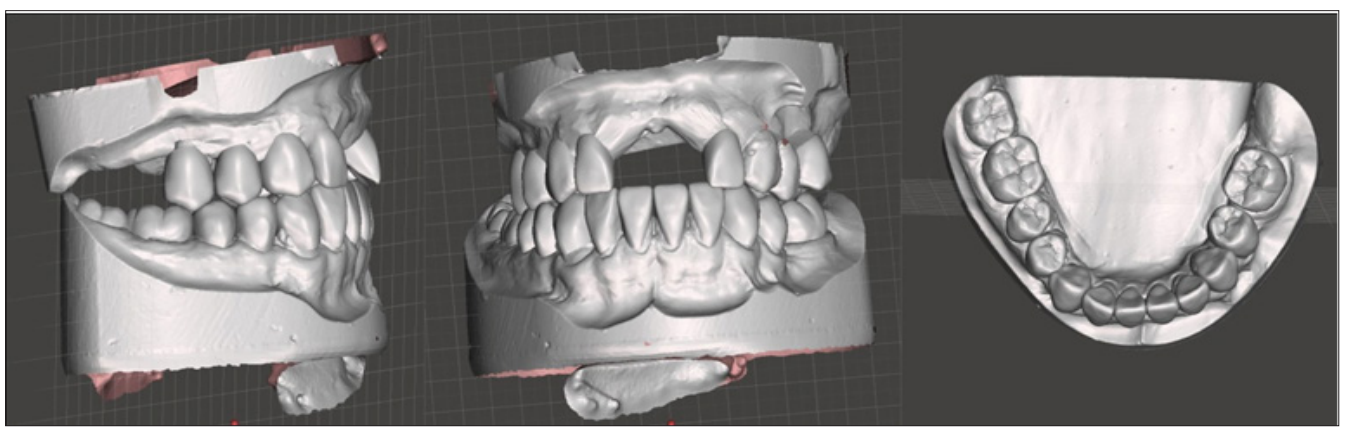

Figure 4: Simulation of orthodontic displacement.

Simulation of Missing Tooth Additions: (Figure 5) It is not in this phase to discuss the type of reconstruction but rather to address the shape of prosthetic teeth. The choice is made to allow both an aesthetic and functional reconstruction. The rules of proportion between the central incisors and the lateral ones are a good guide. In the same way, the width / height proportions of $80 \%$ serve as a basis for work.

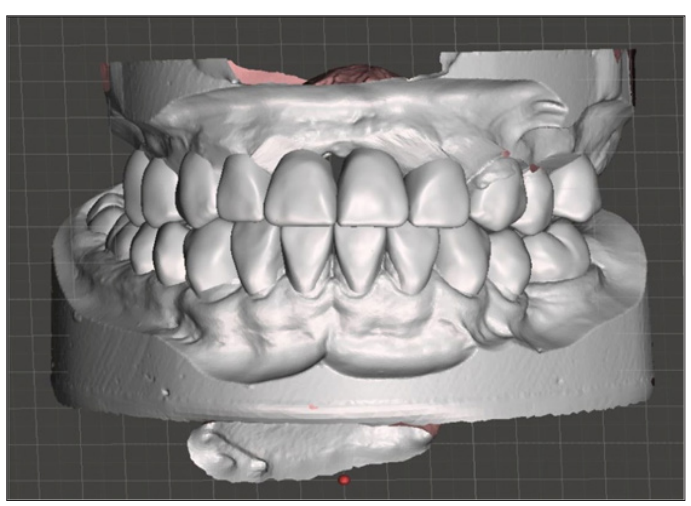

Figure 5: Simulation of missing tooth additions.

\section{Conclusion}

This workflow makes it possible to propose to the patient a visualization of the treatment objectives. This allows having its adherence to a treatment plan that can be long and expensive. The reconstructed dental volumes serve as a basis of reference for the dental movements that will bring the teeth into Class I Angle. In this type of treatment, involving a large-scale rehabilitation, the simulation of treatment allows the various factors involved to become aware of their treatment objectives. This solution seems the best to obtain an aesthetic and functional reconstruction.

\section{References}

1. Hu JCC, Chan HC, Simmer SG, Seymen F, Richardson AS, et al. (2012) Amelogenesis Imperfecta in Two Families with Defined AMELX Deletions in ARHGAP6. Plos One 7(12): 52052.

2. Coffield KD, Phillips C, Brady M, Roberts MW, Strauss RP, et al. (2005) The psychosocial impact of developmental dental defects in people with hereditary amelogenesis imperfecta. J Am Dent Assoc 136(5): 620-630.

3. Strauch S, Hahnel S (2018) Restorative Treatment in Patients with Amelogenesis Imperfecta: A Review. J Prosthodont. 
(C) (i) This work is licensed under Creative Submission Link: https://biomedres.us/submit-manuscript.php

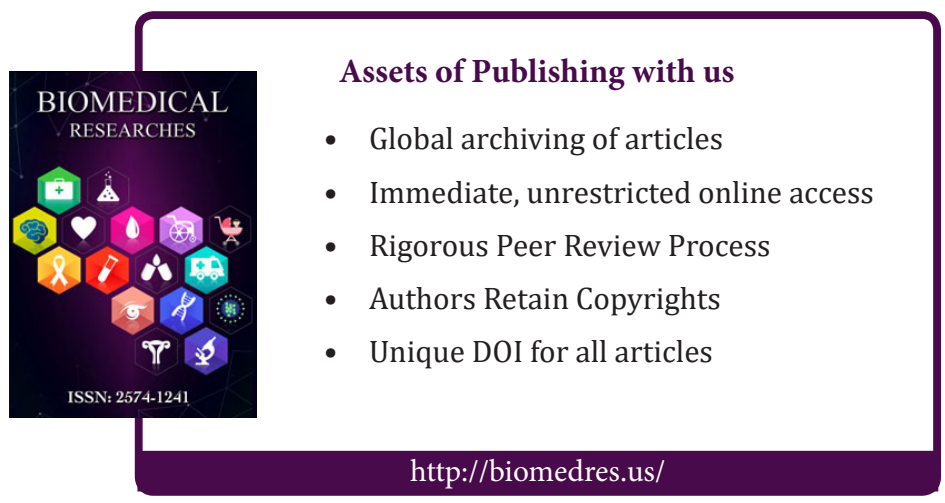

